Pacific Journal of Mathematics

ON THE TOPOLOGY AND GEOMETRY OF ARCWISE
CONNECTED, FINITE-DIMENSIONAL GROUPS 


\title{
ON THE TOPOLOGY AND GEOMETRY OF ARCWISE CONNECTED, FINITE-DIMENSIONAL GROUPS
}

\author{
SIGMUND N. HUDSON
}

\begin{abstract}
If $G$ is an arcwise connected, finite-dimensional group, then it is known that there exists a connected Lie group $G^{\sim}$ and a continuous, one-one, onto homomorphism $i: G^{\sim} \rightarrow$ $G$. Results are obtained for describing the topology of $G$ in terms of the geometry of the manifold $G^{\sim}$ and of $i$. The major result is that there is a closed subgroup $S$ of $G^{\sim}$ and arbitrarily small neighborhoods $U$ at the identity of $G$ such that there are real numbers $r$ and $s$ satisfying $i^{-1}(U)$ is the union of mutually disjoint open balls in $G^{\sim}$ of radius $r$ scattered along the submanifold $S$ in such a manner that the balls are separated by at least $s$. In the case that $G$ is embedded in a locally compact group, more detailed information is given for the distribution of the open balls in $G^{\sim}$.
\end{abstract}

1. Introduction. If $G$ is an arcwise connected, finite-dimensional topological group, then Gleason, Palais, Montgomery, and Zippin have shown that there is a Lie group (connected) $G^{\sim}$ and a continuous, one-one, onto homomorphism $i: G^{\sim} \rightarrow G$. Thereby the study of the algebraic structure of an arcwise connected, finitedimensional group is reduced to that of connected Lie groups. The main interest in this theorem is for the case that $G$ is not locally compact (for in the case of locally compact $G$, it follows that $G$ is a Lie group). In this paper the local and global topological structure of $G$ is studied (assuming $G$ is not locally compact).

One may assume that as sets $G=G^{\sim}$, and the topology of $G^{\sim}$ is obtained by using as a base of open sets the arc components of open subsets in $G$. Also $i: G^{\sim} \rightarrow G$ is the identity function.

To describe the topology of $G$, the geometry of the manifold structure of $G^{\sim}$ serves as a tool. It is shown below that there is a right invariant Riemannian metric $d$ for $G^{\sim}$ and there is a base $\mathscr{B}$ of neighborhoods at $1 \in G$ such that for all $r, n>0$ there is $W \in \mathscr{B}$ and subsets $S$ and $D$ of $G$ with $W=S D$ and with $S \times D$ and $S D$ homeomorphic, where $S$ is an open ball in $G^{\sim}$ of radius less than $r$ with respect to $d$ and where $D$ is a discrete subset of $G^{\sim}$ whose members are separated by at least $n$. This means that no two of them simultaneously belong to any translate of $B^{n}$, where $B$ is the open ball of radius 1 at 1 . Also there is a closed subgroup $L$ of $G$ such that for every $W \in \mathscr{B}$ the arc components $S d$ of $W$ all intersect with $L$. With respect to this property, $L$ is minimal in dimension, and furthermore $L$ is a normal subgroup and $G / L$ is a 
Lie group (see Theorems 1,3, and 4). One could say that each neighborhood in $\mathscr{B}$ is the union of its arc components, where each arc component is an open ball of radius $r$, these components being distributed along the subgroup and submanifold $i^{-1}(L)$ in $G^{\sim}$.

A special case of interest is the case that $G$ is embedded in a locally compact group (see Theorem 2). In this case it is shown that there exists a discrete, abelian subgroup $E$ of $G^{\sim}$ such that every $D$ as given above is contained in $E$. If the locally compact group is Lie, even more detailed information is obtained on $E$.

Examples show that, in the case that $G$ is not embedded as an analytic subgroup of a locally compact group, there may be no such abelian, discrete subgroup $E$ of $G^{\sim}$. In fact, Nienhuys and Rolewicz have constructed a topology for the real numbers $R$ so that $R$ is a topological group with this topology, is arcwise connected, and has only $\{0\}$ and $R$ as closed subgroups. Using modifications of the method of construction for $R$, examples are constructed for a topo$\log y$ for $R^{n}$ so that $\{0\}$ and $R^{n}$ are the only closed subgroups.

In the last section of this paper, a study is made of compact subgroups of $G$ and of the closure of arcwise connected subgroups of $G$. It is shown that $G$ has maximal compact subgroups; in fact, they are $i(K)$, where $i: G^{\sim} \rightarrow G$ and where $K$ is a maximal compact subgroup of $G^{\sim}$. In proving this theorem, it is shown that for each compact subgroup $K$ of $G, i^{-1}(K)$ is compact in $G^{\sim}$. One can ask whether for each compact subset $C$ of $G i^{-1}(C)$ is compact in $G^{\sim}$. An example shows the answer to be no; however it is shown that for each compact arcwise connected subset $A$ of $G, i^{-1}(A)$ is compact in $G^{\sim}$. There are other examples to show that certain known results for one-parameter or for closed subgroups of locally compact groups cannot be generalized to the case of such subgroups of arcwise connected, finite-dimensional groups.

2. Preliminary definitions and results. For a topological group $G$, there is a topological group $G^{\sim}$ obtained from $G$ by using the same algebraic structure for $G^{\sim}$, and defining a topology for $G^{\sim}$ by using as a base for the topology the collection of all arc components of all open subsets of $G$. The identity function $i: G^{\sim} \rightarrow G$ is continuous; and if $G$ is arcwise connected and finite-dimensional, then $G^{\sim}$ is a connected Lie group. Other relationships between $G$ and $G^{\sim}$ are discussed in [4] and [10].

The following two theorems do not seem to be readily available in the literature.

THEOREM. Let $G$ be a compact connected abelian group. Then there is a compact totally disconnected subgroup $D$ of $G$ such that 
$G / D$ is a toral group. A toral group is one isomorphic (as topological groups) with the product of a collection of circle groups, the collection possibly being infinite.

The proof of this theorem uses Pontrjagin duality and a Hausdorff maximality argument.

THeOREM. Let $G$ be arcwise connected and finite-dimensional, with $i: G^{\sim} \rightarrow G$. If $S$ is a closed subgroup of $G$, then $i^{-1}(S)$, as a subgroup of $G^{\sim}$ with the relative topology, is isomorphic to $S^{\sim}$.

Proof. Since $G^{\sim}$ is a connected Lie group, $i^{-1}(S)$ is a Lie group with a countable number of arc components. We define $j_{n}, 1 \leqq n \leqq$ 3 , as the identity function from a set onto itself, where $j_{1}: i^{-1}(S) \rightarrow$ $S^{\sim}$, where $j_{2}: S^{\sim} \rightarrow S$, and $j_{3}: i^{-1}(S) \rightarrow S$. Clearly $j_{2}$ and $j_{3}$ are continuous. Then $j_{1}$ is continuous by $\left[4\right.$, p. 643]. So $j_{1}$ is a continuous, one-one, onto homomorphism from a $\sigma$-compact locally compact group to itself, so it is an isomorphism by [8, p. 7].

By a vector group it is meant a topological group isomorphic to $R^{n}$ for some $n$.

For a connected Lie group $L$, there exists a metric $d$ on $L$ and a real number $t>0$ such that (1) $d$ is a so-called Riemannian metric on $L$ and $d$ is right invariant $(d(y x, z x)=d(y, z)$ for all $x, y, z \in L)$, (2) the neighborhood $N(1, t)=\{x \in L: d(x, 1)<t\}$ is a normal neighborhood of $1 \in L$ with respect to exp: $\mathscr{L}(L) \rightarrow L$ and $d$, and $N(1, t)^{-}$is compact (3) $d(x, y)=\inf \left\{\sum_{i=1}^{k} d\left(z_{i}, z_{i+1}\right): z_{1}=x, z_{k}=y\right.$, and there is a geodesic in $L$ connecting $z_{i}$ to $\left.z_{i+1}\right\}$. Such $d$ are discussed in [6]. We assume without loss of generality that $d$ has been "normalized" so that $t$ (from (2) above) is the real number 1 . This may be done by using a scalar multiple of $d$.

Definition. Let $L$ be a connected Lie group. We say that $L$ has a normalized Riemannian metric $d$ in the case that a metric $d$ with respect to the above properties is used for $L$, including the property that $N(1,1)^{-}$is compact.

3. Main theorems. We begin with a theorem describing the neighborhoods of 1 in an arcwise connected, finite-dimensional group $G$, a theorem of fundamental importance. The theorem says that, with respect to the manifold $G^{\sim}$ and its metric $d$, smaller neighborhoods at $1 \in G$ have arc components which have smaller diameter and which are separated from one another by greater "distances". The quotes are used for the following reason. Customarily one defines subsets $P$ and $Q$ of $G^{\sim}$ to be separated by at least $n$ to mean 
that $d(P, Q)>n$ for a metric $d$ on $G^{\sim}$. For our purposes, this is not appropriate, because $N(1, n)$ may not have compact closure, where $N(1, n)=\left\{x \in G^{\sim}: d(x, 1)<n\right\}$, particularly if $n$ is large enough and if exp: $\mathscr{L}\left(G^{\sim}\right) \rightarrow G^{\sim}$ is not onto. However, if we let $B$ denote $N(1,1)$, which has compact closure by construction of $d$ in $\S 2$, then $B^{n}$ will have compact closure; because $B^{n} \subset\left(B^{-}\right)^{n}$.

Definition. Let $P$ and $Q$ be subsets of a connected Lie group $G^{\sim}$ with normalized Riemannian metric $d$. Define $B$ by $B=N(1,1)$, so that $B$ has compact closure. We say that $P$ and $Q$ are separated by at least $n$ (where $n$ is an even natural number) in the case that for all $x \in G^{\sim}, P$ and $Q$ do not both meet $B^{m} x$, where $m=n / 2$. That is, at least one of $P$ and $Q$ have an empty intersection with $B^{m} x$. Informally, we think of $B^{m}$ as having "width $2 \mathrm{~m}$ " and having "pseudo-radius $m$ ", and we think of $P$ and $Q$ being separated by at least "width $n$ ". In fact, if $G^{\sim}$ is isomorphic to the vector group $R^{k}$, then $d$ may be so chosen that $P$ and $Q$ being separated by at least "width $n$ " is equivalent to $d(P, Q) \geqq n$.

THEOREM 1. Let $G$ be arcwise connected and finite-dimensional, and let $i: G^{\sim} \rightarrow G$, where $G^{\sim}$ has metric $d$ as above. There exists a base $\mathscr{B}$ of neighborhoods at 1 in $G$ such that for any $r, n>0, n$ a natural, even number, there is $U \in \mathscr{B}$ satisfying:

(a) $U$ has a subset $D$ discrete in $G^{\sim}$ such that $U=S D$, where $S$ is an open ball at 1 in $G^{\sim}$ with respect to $d$, where $1 \in D$, and $S \times D \rightarrow S D$ given by multiplication is a homeomorphism,

(b) the arc components of $U$ are $S d$, for $d \in D$, and if $P$ and $Q$ are different arc components of $U$, then radius $(P)<r$, and $P$ and $Q$ are separated by at least $n$,

(c) $D$ is countable, totally disconnected, Hausdorff, hereditarily paracompact, and 0-dimensional.

Proof. Let $V$ be a neighborhood of 1 in $G$, and let $r, n>0$. We consider $G^{\sim}$ as a transformation group acting on the space $G$ via $\pi: G^{\sim} \times G \rightarrow G$ given by $\pi(x, y)=x y$. In as much as $i: G^{\sim} \rightarrow G$ is continuous then $\pi$ is continuous and routine arguments verify that $\left(G^{\sim}, G, \pi\right)$ is a topological transformation group. By the local crosssection theorem (see [9, p. 314]) there exists a compact ball $K$ at $1 \in G^{\sim}$, where $K \subset N(1,1)$ and radius $K=q$, and there exists a closed subset $E \subset G$ with $1 \in E$ such that $K \times E \rightarrow K E$ is a homeomorphism, that $K E$ contains a neighborhood of $1 \in G$, and that $K E \subset V$. We now let $K$ denote the open ball of radius $q$ at $1 \in G^{\sim}$. We see that $E$ contains no $\operatorname{arcs,}$ for otherwise $\operatorname{dim} K E>\operatorname{dim} G=\operatorname{dim} G^{\sim}$. Using the projection $K \times E \rightarrow E$, it is clear that the arcs of $K \times E$ are 
$K \times\{y\}, y \in E$. Since $i^{-1}(K E)$ is open in $G^{\sim}$, and because an open subset of a connected Lie group has a countable number of arc components, we have that $E$ is countable. Since $E$ is a subset of $G, E$ is completely regular; so $E$ contains no connected, nontrivial subsets, and $E$ is totally disconnected.

Next we find an open subset $D$ of $E$ at 1 in $E$ such that for all different $d_{1}, d_{2} \in D$ we have $\left\{d_{1}\right\}$ and $\left\{d_{2}\right\}$ are separated by at least width $n+2$. Suppose by way of contradiction that for all open subsets $D$ of $E$, with $1 \in D$, there exist different $e_{D}$ and $f_{D} \in D$ such that $e_{D}$ and $f_{D}$ are not separated by at least width $n+2$. Therefore, letting $k=(n+2) / 2$, for all $D$ we have that $e_{D}$ and $f_{D}$ belong to $B^{k} z$, that $e_{D}=b_{1} \cdots b_{k} z$, that $f_{D}=c_{1} \cdots c_{k} z$ and that $e_{D} f_{D}^{-1}=$ $b_{1} \cdots b_{k} z z^{-1} c_{k}^{-1} \cdots c_{1}^{-1} \in B^{2 k}$. Consequently, since $B^{2 k}$ has compact closure in $G^{\sim}, e_{D} f_{D}^{-1}$ has a convergent subnet in $G^{\sim}$ converging to some $w \in G^{\sim}$. This subnet we also call by the same name. Note that $w \neq 1$, because $e_{D}$ and $f_{D}$ belong to different open balls $K e_{D}$ and $K f_{D}$ of $K E$ for all $D$, making $d\left(e_{D}, f_{D}\right)>q$. Then $d\left(e_{D} f_{D}^{-1}, 1\right)>q$, implying that $d(w, 1) \geqq q$ and $w \neq 1$. On the other hand, because $i: G^{\sim} \rightarrow$ $G$ is continuous, $i\left(e_{D} f_{D}^{-1}\right)$ converges to $i(w)$ in $G$. In $G$ the nets $e_{D}$ and $f_{D}$ both converge to 1 , so the net $e_{D} f_{D}^{-1}$ converges to both 1 and $w$, a contradiction. So there is a desired subset $D$.

Next we define $S=N(1, t)$, where $t=\min \{r / 2, q\}$. Recall that $q<1$. Next we show that $S d_{1}$ and $S d_{2}$ are separated by at least width $n$. If there should exist $z$ such that $S d_{1}$ and $S d_{2}$ both meet $B^{k} z(k=n / 2)$, then $u \in S d_{1} \cap B^{k} z \subset B d_{1}$ and $v \in S d_{2} \cap B^{k} z \subset B d_{2}$, and $d_{1} \in B u \subset B B^{k} z$, also $d_{2} \in B v \subset B B^{k} z$, so $d_{1}$ and $d_{2}$ are not separated by at least $2(k+1)=2(n / 2+1)=n+2$. Therefore $S d_{1}$ and $S d_{2}$ are separated by at least $n$.

We have established (a) and (b) of the theorem. To show (c), we have that every subset of $D$ is countable, hence Lindelöf. So every subset is paracompact [3, p. 174]. So $D$ is hereditarily paracompact. In as much as $D$ is totally disconnected, every compact subset $F$ of $D$ has cohomological dimension $c d F$ equal to $0[1, \mathrm{p} .218]$, [10]; so $c d D=0$. Note that the inductive dimension of $D$ is also $0[2$, p. 279].

Next we prove in Theorem 2 that if the arcwise connected, finite-dimensional $G$ is contained in a locally compact group $L$, then the structure of the $D$ in the previous theorem is improved; in fact, there exists a subgroup $E$ of $G^{\sim}$ isomorphic to $Z^{k}$ such that for every $U=S D$ of the previous theorem we have $D \subset E$. The closure of $E$ in $L$ is a compact group. If furthermore $L$ is a Lie group, then the closure of $E$ in $L$ is a torus subgroup of $L$, as shown by the next Theorem 2. We first present two lemmae. 
LEMMA 1. Let $L$ be a compact group, let $G$ be a subgroup of $L$ such that $G^{\sim}$ is isomorphic to $R^{n}$ and such that $G$ is dense in $L$, and let $C$ be a compact subgroup of $L$ with $L=G C$. Then there exists a compact subgroup $K \subset C$ such that $G^{-}=G K$, that $(G \cap K)^{-}=K$, and $i^{-1}(G \cap K)$ is discrete in $G^{\sim}$.

Proof. Note that $G$ and $L$ are abelian. We let $D$ be a closed, totally disconnected subgroup of the component $J$ at 1 in $C$ such that $J / D$ is a toral group (possibly infinite-dimensional). We let $\varphi$ : $G C \rightarrow G C / D$ be the coset homomorphism, with $\varphi(G)=G_{1}, \varphi(J)=J_{1}$, and $\varphi(C)=C_{1}$. Then $G_{1} C_{1}$ has the same properties as $G C$, except that the identity component $J_{1}$ at 1 in $C_{1}$ is a toral group. This implies that $J_{1}$ is a direct factor of $C_{1}$ [7, p. 419], so that there exists a subgroup $E$ of $C_{1}$ such that $C_{1}=E \otimes J_{1}$. Clearly $E$ is totally disconnected. We let $\pi: G_{1} C_{1} \rightarrow G_{1} C_{1} / E$ be the coset homomorphism with $\pi\left(G_{1}\right)=G_{2}$, with $\pi\left(C_{1}\right)=C_{2}$. Then $G_{2} C_{2}$ satisfies the same properties as $G_{1} C_{1}$ and $G C$, except $C_{2}$ is a toral group, whereas $C_{1}$ or possibly $C$ is not a toral group. We now prove Lemma 1 for $G_{2} C_{2}$, rather than $G C$; and Lemma 2 below, applied to $G_{1} C_{1}$ and $G_{2} C_{2}$, then next applied to $G C$ and $G_{1} C_{1}$, will finish the proof of Lemma 1. Therefore we may assume without loss of generality that $C$ is a toral subgroup of $L=G C$ ( $C$ possibly infinite-dimensional). In the closed subgroup $i^{-1}(G \cap C)$ of $G^{\sim}$, we let $V$ denote the component of $i^{-1}(G \cap C)$ containing 1 . Then $V$ is a vector group in $G^{\sim}$. Using [5, p. 164] we may find a neighborhood $U$ of 1 in $V^{\sim}$ and a toral subgroup $K$ of the toral group $C$ such that $U K$ is a neighborhood of 1 in $C$ and such that $U \times K \rightarrow U K$ is a homeomorphism and a local homomorphism. The countability assumptions of [5] are satisfied in the setting of Lemma 1, Lemma 2, and Theorem 2 , by using the fact that $i\left(G^{\sim}\right)$ is dense in $L$. We will prove that $K$ is the desired subgroup. If $i^{-1}(G \cap K)$ should contain a one-parameter subgroup $P$, then $U \cap K$ contains $V \cap P$, a contradiction. Next, to show $G K=G C$, we have that $G K=G V K=G C$. Finally to prove that $(G \cap K)^{-}=K$, we denote $(G \cap K)^{-}$by $F$, and establish that $G F$ is a compact subgroup of $G C$. To do this, we consider $G F / F$. This group is arcwise connected and finite-dimensional. Also $\varphi: G F / F \rightarrow G K / K$ defined by $\varphi(x F)=x K$ is a one-one, onto homomorphism, using that $F \subset K$ and the definition of $F$. Since $G K / K$ is a torus group and since $(G F / F)^{\sim}$ is a Lie group, it is clear that $\varphi$ is an isomorphism. So $G F / F$ is compact. Since $F$ is compact, we have that $G F$ is compact. Because $G F$ is a compact subgroup containing the dense $G$, we have that $G F=G C=G K$. We use the equality $G F=G K$ to prove that $F=K$. Already $F \subset K$, so let $x \in K$. Then $x \in G F$, so $x=g c$, where $g \in G$ and $c \in(G \cap K)^{-}$. So 
$c \in K$, so $g \in K$, so $g \in G \cap K$, so $g \in F$, and $x \in F$. So $K \subset F$. So Lemma 1 is proved. We note that if $L$ is a Lie group, then $K$ can be chosen to be a torus subgroup of $L$.

LEMMA 2. Let $\alpha: G K \rightarrow H M$ be an onto homomorphism of compact abelian groups, with the kernal of $\alpha$ being totally disconnected and contained in $K$, with $\alpha(G)=H$ and $\alpha(K)=M$, with $G^{\sim}$ and $H^{\sim}$ isomorphic to $R^{n}$, and with $G$ and $H$ dense in $G K$ and $H M$ respectively. If $H M$ satisfies the conclusion of Lemma 1, then GK satisfies the conclusion of Lemma 1.

Proof. By hypothesis, there is a compact subgroup $M_{1} \subset M$ such that $H^{-}=H M_{1}$, etc. One defines $C$ by $C=\alpha^{-1}\left(M_{1}\right)$. Then arguments such as used at the end of Lemma 1 show that $C \subset K$, that $G^{-}=G C$, etc.

THeORem 2. Let $G$ be arcwise connected and finite-dimensional, and suppose that $G$ is a dense subgroup of the locally compact group $L$. Then there exist $\mathscr{B}$ and $d$ as in Theorem 1, and there exists an abelian compact subgroup $K$, with $K \subset Z(L) \cdot R$, for $R=$ the radical of $L$ and $Z(L)=$ the center of $L$, such that for any $r, n>0$, there exists $U \in \mathscr{B}$ satisfying:

(a) letting $A$ denote the subgroup $G \cap K$, the neighborhood $U$ has a subset $D$, with $D \subset A$, such that $D$ is discrete in $G^{\sim}$ and that $U=S D$, where $S$ is an open ball at $1 \in G^{\sim}$ with respect to $d$, where $1 \in D$, and where $S \times D \rightarrow S D$ is a homeomorphism,

(b) the arc components of $U$ are $S d$ for $d \in D$; and, if $P$ and $Q$ are different are components of $U$, then radius $(P)<r$ and $P$ and $Q$ are separated by at least width $n$,

(c) $A$ is a countable subgroup of $G$, discrete in $G^{\sim}$ and totally disconnected in $G$, and the closure of $A$ in $L$ is $K$,

(d) $G^{-}=G K$ and $G$ is a normal subgroup of $G^{-}$. If $L$ is a Lie group, then $K$ is a torus subgroup of $G^{-}$.

Proof. The proof consists of the cases for $G$ abelian, for $G$ solvable, for $G$ semisimple, and then the general case.

In the case that $G^{\sim}$ is abelian, we have that $L$ is abelian. It is known that there are subgroups $B_{2}, B_{3}, B_{4}$ of $G$ such that $G=$ $B_{2} B_{3} B_{4}$, where $B_{4}$ is a vector subgroup of $G^{\sim}$, of $G$, and of $L$, and where $B_{2}$ is the maximal compact subgroup of $G^{\sim}$, and where $B_{3}$ is a vector subgroup of $G^{\sim}$ such that every one-parameter subgroup of $B_{3}$ is nonclosed in $L$, and where $G^{\sim}$ is the direct product of the $B_{i}$. It follows that $G^{-}=B_{2} B_{3}^{-} B_{4}$. Using the known facts about the product structure of locally compact, connected, abelian groups [7, 
pp. 90,95] we may assume that $G=B_{3}$, in as much as $B_{2}$ and $B_{4}$ are direct factors in $G$ and $L$. We now have that $L=B_{3} B_{2}=G B_{2}$, where $G$ is dense in $L$ and where $G^{\sim}$ is isomorphic to $R^{n}$, so that Lemma 1 is applicable. Therefore, the major part of the abelian case of Theorem 2 has been established, and we omit the remaining parts of the abelian case.

Next assume that $G^{\sim}$ is semisimple. To give an example of this situation, let $H$ be a connected semisimple Lie group such that there exists $d \in Z(H)$ with the group $D$ generated by $d$ isomorphic to the integers (and discrete in $H$ ). Let $K$ be a compact, monothetic group with the powers of $e$ dense in $K$ (here $K$ may possibly be disconnected or may be infinite-dimensional). In $H \times K$ let $D^{*}$ be $\left\{(d, e)^{n}: n\right.$ is an integer $\}$. Note that $D^{*}$ is discrete and central in $H \times K$. Letting $\varphi: H \times K \rightarrow(H \times K) / D^{*}$ be the coset homomorphism, we have that $\varphi(H)$ is dense in the locally compact $(H \times K) /$ $D^{*}$ and that $\varphi(H)^{\sim}$ is isomorphic with $H$. Continuing with the the proof, we let $\left\{d_{1}, \cdots, d_{t}\right\}$ be a set of generators for the finitely generated, central subgroup $Z(G)$, such that $\left\{d_{1}, \cdots, d_{k}\right\}$ generates a discrete subgroup of $L$, and each element of $\left\{d_{k+1}, \cdots, d_{t}\right\}$ generates a nondiscrete subgroup of $L$. If we let $\Gamma(x)$ denote the closure of the group generated by $x$, then Weil's lemma shows that $\Gamma\left(x_{i}\right)$ is compact for $k+1 \leqq j \leqq t[7, \mathrm{p}$. 84]. We let $R$ be the radical of $L$, and one proves that every element of $R$ is the limit of elements from the group $D$ generated by $\left\{d_{k+1}, \cdots, d_{t}\right\}$. Furthermore, it can be seen that $R$ is the connected component of $D^{-}$containing 1 . If we define $K$ to be $D^{-}$, if we let $S$ be an open ball at $1 \in G^{\sim}$ such that $S^{2} \cap Z\left(G^{\sim}\right)=\{1\}$, then it can be shown that $S \times D^{-} \rightarrow S D^{-}$is a homeomorphism, and the other conclusions of the theorem follow readily. If $L$ is a Lie group, it is clear that $D^{-}=R$ is a torus subgroup of $L$.

We omit the proof of the solvable case of the theorem, since the methods of proof are similar to those in the other cases.

In the general case, we let $R$ denote the radical of $G^{\sim}$, we let $S$ be a maximal semisimple analytic subgroup of $G^{\sim}$ such that $G^{\sim}=$ $S R$, and we let $P$ be the radical of $L$. It is easily shown that $R \subset$ $P$. Since $R$ and $S$ are arcwise connected and finite-dimensional, we may assume using the solvable and semisimple cases for the theorem that there exists a compact abelian subgroup $K_{2} \subset R^{-} \subset L$ (given by the solvable case) and a compact subgroup $K_{1} \subset Z(L) \subset S^{-} \subset L$ (given the semisimple case), satisfying the appropriate conditions. (If $L$ is a Lie group, then $K_{1}$ and $K_{2}$ are torus subgroups.) It is tempting to define $A$ and $K=A^{-}$by letting $A^{-}=K_{1} K_{2}$, but it may happen that $K_{1} \cap R$ and possibly $K_{1} K_{2} \cap G$ contain a nontrivial oneparameter subgroup, an undesirable property if we wish $K \cap G$ to 
be discrete in $G^{\sim}$. So we "rechoose" as follows. The purpose of the following discussion is to obtain an appropriate subgroup of $K_{1} K_{2}$. We have that $K_{1} K_{2} \cap G$ is closed in $G$, and so $K_{1} K_{2} \cap G$ is closed in $G^{\sim}$. So the identity arc component of $i^{-1}\left(K_{1} K_{2} \cap G\right)$, denoted by $E$, is a closed vector subgroup of $i^{-1}\left(K_{1} K_{2} \cap G\right)$. Since $E$ is a vector subgroup of $K_{1} K_{2}$, there exists a neighborhood $U$ of 1 in $E^{\sim}$ and a compact subgroup $K$ of $K_{1} K_{2}$ such that $U K$ is a neighborhood of 1 in $K_{1} K_{2}$ and $U \times K \rightarrow U K$ is a homeomorphism and local homomorphism. We obtain such $U$ and $K$ by using a compact, totally disconnected subgroup $D$ of $K_{1} K_{2}$ such that $K_{1} K_{2} / D$ is a toral group, by using [5, p. 164], and by using the above Lemma 2 . We will prove that $K$ is the desired subgroup of $L$. Note that $K \subset Z(L) P$. Further note that if $L$ were a Lie group, then $K_{1}, K_{2}$, and $K$ would be torus subgroups of $L$.

We prove first that $G \cap K$ is dense in $K$. For any $x \in K$, and any $V$ open in $K$ containing $x$, we have that $U V$ is open in $U K$, where $U$ is as above. Since $G \cap K_{1} K_{2}$ is dense in $K_{1} K_{2}$, there exists $g \in G \cap U V$. Because $g=u v$ and $g$ and $u$ belong to $G$, we have that $v \in G$. So $v \in G \cap V$, and $G \cap K$ is dense in $K$. Next we show that $G \cap K$ is discrete in $G^{\sim}$. If $V$ is a one-parameter subgroup of $G \cap K$, then $V \subset E$. Since $E \cap K$ is discrete in $E^{\sim}$, we have that $V=\{1\}$. Finally we show that $G K=L$. Using normality of $G$ in $L$ (a well-known result, for example see [5]), it follows that $G K$ is a subgroup of $L$. Next we show that $G K$ contains a neighborhood in $L$, and connectedness of $L$ will imply that $G K=L$. We have that $G K \supset G U K \supset G W$, where $W$ is a neighborhood in $K_{1} K_{2}$. But $G W$ clearly contains a neighborhood in $S K_{1} R K_{2}=L$.

The proof of Theorem 2 is now easily finished by using Theorem 1 .

REMARKS ON THEOREM 2. Several authors, particularly Goto, have proved results similar to Theorem 2. Their results are not as detailed about $K$ as the present Theorem 2. The subgroup $K$ must satisfy several competing conditions. First, it must be "large enough", because $G K$ must be all of $L$. Next it must be "small enough", because $K$ must satisfy that $G \cap K$ is discrete. Finally $K$ must be "appropriately orthogonal to a neighborhood in $G$," because $K$ must satisfy that $G \cap K$ is dense in $K$.

Next we show that for an arcwise connected, finite-dimensional group $G$, there is a subgroup $S$, closed in $G$ and $G^{\sim}$, such that arbitrarily small open sets at 1 in $G$ have all of their arc components concentrated on $S$ and that $G / S$ is an analytic manifold.

Definition $\mathscr{S}$. Let $G$ be arcwise connected, finite-dimensional, 
with $i: G^{\sim} \rightarrow G$. We let $\mathscr{S}$ be the collection of all subgroups $S$ of $G$ satisfying (1) $i^{-1}(S)$ is a closed subgroup of $G^{\sim}$, and (2) there exists a neighborhood base $\mathscr{B}$ at $1 \in G$ of open sets in $G$ such that for all $B \in \mathscr{B}$ and for any arc component $A$ of $B$, we have $A \cap S \neq \varnothing$.

THEOREM 3. Let $G$ be arcwise connected and finite-dimensional, with $i: G^{\sim} \rightarrow G$. (i) If $R$ and $S \in \mathscr{S}$, then $R \cap S \in \mathscr{S}$. (ii) Every $S \in \mathscr{S}$ is a closed subgroup of $G$. (iii) $\mathscr{S}$ is nonempty, because $G \in \mathscr{S}$. (iv) $G^{\sim} / i^{-1}(S)$ and $G / S$ are homeomorphic coset spaces, so that $G / S$ is an analytic manifold. (v) If $S \in \mathscr{S}$ and $x \in G$, then $x S x^{-1} \in \mathscr{S}$.

Proof. (i) Clearly condition (1) of the definition of $\mathscr{S}$ is satisfied by $R \cap S$. To prove (2) of the definition, let $\mathscr{D}$ be a neighborhood base for $R$ and $\mathscr{E}$ a neighborhood base for $S$, each satisfying (2). In order to construct a neighborhood base $\mathscr{C}$ for $R \cap S$, let $U$ be any neighborhood of 1 in $G$. Choose $W$ a neighborhood of 1 in $G$ such that $W^{3} \subset U$ and such that the arc components of $W$ are open balls of radius $r>0$, using Theorem 1. There is a $D \in \mathscr{D}$ such that $D \subset W$, and there is an $E \in \mathscr{E}$ such that $E \subset D$. We define $B$ to be the open ball of radius $r$ at 1 (so that $B \subset W$ ), and we define $C=B B E$. Then $C$ is a neighborhood of 1 in $G$, and $C$ is a candidate for $\mathscr{C}$. It is clear that $C \subset U$, because $B B E \subset$ $W^{3} \subset U$. In order to show that every arc component of $C$ meets $R \cap S$, we let $A$ be some arc component of $B B E$. Then be belongs to $A$ for some $b \in B B$ and some $e \in E$. Then $e$ is contained in some arc component $E_{e}$ of the neighborhood $E$, and the arc component $E_{e}$ satisfies $E_{e} \cap S \neq \varnothing$. Therefore $A \cap S \neq \varnothing$. Furthermore, $E_{e}$ is contained in some arc component $D_{e}$ of $D$. We will show that $D_{e} \subset A$. It is sufficient to show that $D_{e} \subset B B E_{e}$. Let $x \in D_{e}$. Since $x$ and $e$ are both in the same arc component $D_{e}$, which is contained in $W$, there is an open ball of $W$, whose center is $y$, containing $x$ and $e$. Since $d(y, x)<r$, we have $x \in B y$; and since $d(y, e)<r$, we have $y \in B e$. So $x \in B B e \subset B B E_{e}$. So we now have that $A \cap R \neq \varnothing$, in as much as $D_{e} \cap R \neq \varnothing$. So the arc component $A$ of $B B E$ meets $R \cap S$. So such a neighborhood base $\mathscr{C}$ exists as claimed.

(ii) Let $S \in \mathscr{S}$. If $x \in G$, and $x \notin S$, for a Riemannian metric $d$ given by above, we have that $d\left(x^{-1}, S\right)>0$, since $i^{-1}(S)$ is a closed subgroup of $G^{\sim}$. Again by Theorem 1, let $U$ be a neighborhood of 1 in $G$ such that every arc component of $U$ has diameter less than $d\left(x^{-1}, S\right)$, and let $V \subset U$, where $V$ is a neighborhood of 1 in $G$ with every arc component of $V$ meeting $S$. Suppose by way of contradiction that $x V \cap S \neq \varnothing$. Let $s \in x V \cap S$; then $s \in x A$, where $A$ is 
some arc component of $V$. Then $x^{-1} s \in A$, and furthermore there is an element $a \in A \cap S$. Hence both $x^{-1} s$ and $a$ belong to $A$. Consequently $d\left(x^{-1} s, \alpha\right)<\operatorname{diam} A$, so that $d\left(x^{-1}, a s^{-1}\right)$ as well as $d\left(x^{-1}, S\right)$ are less than $\operatorname{diam} A$. But $A \subset V \subset U$, and we recall that an arc component $A$ of $V$ satisfies $\operatorname{diam} A<d\left(x^{-1}, S\right)$, a contradiction. So we have that $x V \cap S=\varnothing$. Hence $S$ is a closed subgroup of $G$.

To prove (iv), we have the following commutative diagram:

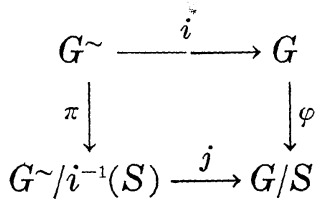

where $\pi$ and $\varphi$ are the coset mappings, and $j(x S)=x S$. Then $j$ is clearly continuous, one-one, and onto. To show $j$ is open, since $G^{\sim} / i^{-1}(S)$ and its image are homogeneous, it is sufficient to prove $j$ is open at $i^{-1}(S) \in G^{\sim} / i^{-1}(S)$. Let $U i^{-1}(S)$ be an open set at $i^{-1}(S)$, where $U$ is open at $1 \in G^{\sim}$. Then there exists an open ball $B \subset U$, with $1 \in B$, the radius of $B$ is $r>0$, and $B S \subset U S$. Then there exists an open set $W$ in $G$ at 1 such that every arc component of $W$ has nonempty intersection with $S$ and such that the arc components of $W$ have diameter less than $r / 2$, using Theorem 1 and that for $S$ the definition above gives us a base of neighborhoods. Then routine arguments prove that $W S \subset B S \subset U S$.

Definition. For a topological group $H$, the component of the identity 1 in $H$ will be denoted by $H_{0}$, and the arc component of the identity will be denoted by $H_{a}$. So $H_{a} \subset H_{0}$.

THEOREM 4. Let $G$ be arcwise connected and finite-dimensional, and let $\mathscr{S}$ be as in Definition $S$. Let $S \in \mathscr{S}$ be minimal among elements of $\mathscr{S}$ with respect to $\operatorname{dim} i^{-1}\left(S_{a}\right)$. Then $i^{-1}\left(S_{a}\right)$ is a closed normal subgroup of $G^{\sim}$. If $T$ is another member of $\mathscr{S}$ satisfying $\operatorname{dim} i^{-1}\left(T_{a}\right)=\operatorname{dim} i^{-1}\left(S_{a}\right)$, then $T_{a}=S_{a}$. If $S$ is arcwise connected, then for every $T \in \mathscr{S}, S \subset T$.

Remarks on Theorem 4. If $G$ is a Lie group, then $\{1\}$ is the minimal member of $\mathscr{S}$. Examples in the next section show that if $G$ is non-Lie, then $G$ may be the only member of $\mathscr{S}$. Other examples in the next section show that $S$ may have any dimension between 0 and the dimension of $G^{\sim}$. Theorem 2 above shows that a minimal member of $\mathscr{S}$ with respect to dimension must have dimension zero whenever $G$ is embedded in a locally compact group.

Proof of Theorem 4. Since $i^{-1}(S)$ is a closed subgroup of $G^{\sim}$, 
we have that $i^{-1}(S)_{0}=i^{-1}\left(S_{a}\right)$ is a closed subgroup of $G^{\sim}$. Next let $x \in G$. By Theorem $3, x S x^{-1} \in \mathscr{S}$, and $S \cap x S x^{-1} \in \mathscr{S}$. So $S \cap x S x^{-1}$ has the same dimension as $S$, since $S$ was minimal. Using standard facts about Lie groups and their closed, connected subgroups having the same dimension, it follows that $x\left(i^{-1}(S)_{0}\right) x^{-1}=i^{-1}(S)_{0}$, so that $i^{-1}(S)_{0}$ is a normal subgroup of $G^{\sim}$. If $T$ is another member of $\mathscr{S}$ as in the theorem, then the same type of argument proves that $i^{-1}(S)_{0}=i^{-1}(T)_{0}$. If $S$ is arcwise connected, and if $T \in \mathscr{S}$, then $S=$ $S_{a}=T_{a} \subset T$.

COROLlary. If $G$ is additionally abelian, then there exists $S \in \mathscr{S}$ such that $S$ is minimal with respect to $\operatorname{dim} i^{-1}\left(S_{a}\right)$ and with respect to the number of independent generators of $i^{-1}(S) / i^{-1}\left(S_{a}\right)$. For any $T \in \mathscr{S}$ also minimal in these respects, $S / S \cap T$ and $T / S \cap$ $T$ are finite abelian groups, that is $S$ and $T$ are commensurable in the sense of the theory of discrete subgroups of Lie groups.

Proof. Clearly one can find a subgroup $S \in \mathscr{S}$ such that $S_{a}$ has minimal dimension. Since $S / S_{a}$ is a discrete subgroup of the analytic abelian group $G^{\sim} / S_{a}^{\sim}$, it is a finitely generated, abelian group; so that one may choose $S \in \mathscr{S}$ so that additionally $S / S_{a}$ has a least number of generators. Then $S$ satisfies the required conditions.

The proof of this corollary raises questions for the general case, where $G$ may be nonabelian. The goal is to obtain a "minimal" member of $\mathscr{S}$, where " $S$ is minimal" means that $S \in \mathscr{S}$ is minimal with respect to the two conditions of the previous corollary. For example, in the case for $G$ nonabelian, is the discrete group $S / S_{a}$ a finitely generated subgroup of $G^{\sim} / S_{a}^{\sim}$ ?

4. Arcwise connected subgroups and examples. In this section there are results and examples which have been motivated by theorems about certain subgroups of locally compact groups. In the next two theorems, we study the compact subgroups of an arcwise connected, finite-dimensional group $G$ and how they are related to the compact subgroups of $G^{\sim}$. The next theorem describes a curious situation for the preimages of compact subsets of $G$ with respect to $i: G^{\sim} \rightarrow G$.

THEOREM 5. Let $G$ be arcwise connected and finite-diminisional, and let $i: G^{\sim} \rightarrow G$.

(a) If $K$ is a compact subgroup of $G$, then $i^{-1}(K)$ is compact in $G^{\sim}$.

(b) If $K$ is a compact subset of $G$, then $i^{-1}(K)$ may not be 
compact in $G^{\sim}$.

(c) If $K$ is a compact, arcwise connected subset of $G$, then $i^{-1}(K)$ is a compact subset of $G^{\sim}$.

Proof. (a) We have that $i^{-1}(K)$ is a closed subgroup of the connected Lie group $G^{\sim}$. Hence $i^{-1}(K)$ is a Lie group with a countable number of arc components. Hence by [7, p. 42], $i$ is an open homomorphism, and $K$ and $i^{-1}(K)$ are isomorphic by $i$. Hence $i^{-1}(K)$ is compact.

(b) Let $G$ be the arc component at 1 of a one-dimensional compact, solenoidal group, so that $G^{\sim}$ is isomorphic to the real numbers $R$ with its usual topology. It is known that $G$ is arcwise connected, one-dimensional, and has a sequence $\left\{x_{n}\right\}$ converging to 1 in $G$, such that $i^{-1}\left(\left\{x_{n}\right\}\right)$ is an unbounded sequence in $i^{-1}(G)$. Hence $K=\{1\} \cup\left\{x_{n}\right\}$ is the desired compact subset of $G$.

(c) Our first goal is to construct a certain cover of $G$ (not $K$ ) which has no finite subcover. We present several lemmae:

LEMMA 3. Let $U$ be a neighborhood of $1 \in G$ such that (a) the arc components of $U$ each have diameter less than $r$, where $r<1$, (b) the arc components of $U$ are separated by at least width 4, using a normalized Riemannian metric and the terminology from $\S 2$. Let $V$ be a neighborhood of $1 \in G$, with $V \subset U$, and assume the arc components of $V$ are separated by at least width 4. Define $W$ by $W=\cup\{A: A$ is an arc component of $U$ and $A \cap V \neq \varnothing\}$. Then $W$ is a neighborhood of $1 \in G$.

Proof. Define $Y=B V$, where $B$ is the open ball at $1 \in G^{\sim}$ of radius $r$, that is $B=N(1, r)$. Then $Y$ is open in $G$. The proof will be finished when it is shown that $B V \cap U=U\{A: A$ is an arc component of $U$ and $A \cap U \neq \varnothing\}$. If $V_{1}$ and $V_{2}$ are two arc components of $V$, then routine arguments show that $B V_{1}$ and $B V_{2}$ are separated by at least width 2 . This implies that $B V \cap U$ is contained in the above union. Next, let $x$ belong to the union; that is, $x \in A$ where $A$ is an arc component of $U$ and $A \cap U \neq \varnothing$. Let $P$ be the arc component of $V$ contained in $A$. There exists $y \in P$ with $d(x, y)<1$, since $\operatorname{diam} A<1$. Therefore $x \in B y \subset B P \subset B V$. So $x \in$ $B V \cap U$, and the union is contained in $B V \cap U$, ending the proof.

For each natural number $n$, choose $U_{n}$ such that (1) $U_{n}$ is a neighborhood of $1 \in G$ and $U_{n+1} \subset U_{n}$, (2) the arc components of $U_{1}$ are balls with radius less than 1 , and (3) the arc components of $U_{n}$ are separated by at least width $4^{n+1}$. Define $B$ to be the ball of radius 1 in $G^{\sim}$ at 1 , so that $B^{-}$is compact. Define $W_{1}=U_{1}$, and define $W_{n}=\left(B^{n-1} \cdot \cup\left\{A: A\right.\right.$ is an arc component of $U_{1}$ and $A \cap U_{n} \neq$ 
$\varnothing\}) \cup W_{n-1}$. In other words, $W_{n}$ is the union of $W_{n-1}$ with $B^{n-1} V_{n}$, where $V_{n}=\cup\left\{A: A\right.$ is an arc component of $U_{1}$ and $\left.A \cap U_{n} \neq \varnothing\right\}$. The previous lemma may be used to show that $W_{n}$ is a neighborhood of $1 \in G$. So, informally, $W_{1}$ is the union of open balls of radius less than 1 and separated by at least width 16 ; and $W_{2}$ is the union of a certain subcollection of the open balls from $W_{1}$ (this subcollection determined by $U_{2}$ ), each such ball "expanded by $B$," members of this subcollection separated by at least width 64 , unioned with the remaining open balls of $W_{1}$. We call the $B \cdot A$, such that $A$ is an arc component of $U_{1}$ with $A \cap U_{2} \neq \varnothing$, by the name "the building blocks of $W_{2}$ ". In general, we obtain $W_{n+1}$ from $W_{n}$ by using $U_{n+1}$ to determine a certain subcollection of building blocks $B^{n-1} A$ from $W_{n}$. We then form $B \cdot B^{n-1} A=B^{n} A$, obtaining the building blocks for $W_{n+1}$, so that $W_{n+1}$ is the union of these building blocks $B^{n} A$ with all the previously determined building blocks in $W_{n}, W_{n-1}$, etc. We note that for all $n, W_{n} \subset W_{n+1}$, and each $W_{n}$ is the union of sets of the type $B^{k} A$, where $0 \leqq k \leqq n-1$, and where $A$ is an arc component of $U_{1}$. Of course, $B^{0}=\{1\}$. We remark that for $n$ small, the arc components of $W_{n}$ are just the building blocks $B^{n-1} A$ of $W_{n}$, but when $n$ becomes larger, an arc component of $W_{n}$ may be the union of several building blocks.

We note that $\left\{W_{n}\right\}$ forms an open cover of $G$, because $\left\{B^{n}\right\}$ covers the Lie group $G^{\sim}$, and $B^{n} \subset W_{n+1}$. It is our next goal to study the arc components of $W_{n}$. The next lemma says that the arc components of $W_{n}$ cannot be too large.

LEMMA 4. For each $n$, there is no arc component of $W_{n}$ which contains two or more building blocks obtained at stage $n$, that is, having the form $B^{n-1} A$, where $A$ is an arc component of $U_{1}$.

Proof. For $n=1$, the lemma is clear, because $W_{1}=U_{1}$. Suppose the lemma is true for all $j<n$. By way of contradiction, suppose $B^{n-1} A_{1}$ and $B^{n-1} A_{2}$ are contained in an arc component of $W_{n}$. We may suppose that there is a collection of building blocks $\left\{C_{1}, \cdots, C_{m}\right\}$ satisfying $C_{1}=B^{n-1} A_{1}, C_{m}=B^{n-1} A_{2}, C_{i} \cap C_{i+1} \neq \varnothing$, and each $C_{i}$ is of the form $B^{k-1} A$, for $1 \leqq k<n$, where each $B^{k-1} A$ is a building block from stage $k<n$. In other words, there is a finite sequence of building blocks connecting $B^{n-1} A_{1}$ with $B^{n-1} A_{2}$, all of these in-between building blocks obtained at a stage $k<n$. Let $C_{i_{1}}$ be a building block obtained at level $k$, where $k$ is as large as possible and $k<n$ (so $i_{1} \neq 1, i_{1} \neq m$ ). If there were two such $C_{i_{1}}$, then we would have obtained a finite sequence of building blocks at stage $k<n$, thereby contradicting the induction hypothesis. Next we let $C_{i_{2}}$ be a building block obtained at a stage $j$, where 
$j$ is as large as possible and $j<k<n$. There can be at most two such $C_{i_{2}}$; for if there existed three such, at least two of them would have subscripts $i_{2}$ and $i_{3}$ satisfying $i_{2}$ and $i_{3}$ are between 1 and $i_{1}$, or else $i_{2}$ and $i_{3}$ are between $i_{1}$ and $m$. In either case the sequence from $C_{i_{2}}$ to $C_{i_{3}}$ would be a sequence connecting $C_{i_{2}}$ and $C_{i_{3}}$ at stage $j<n$, again a contradiction to the induction hypothesis. Consequently there are at most two such, say $C_{i_{2}}$ and $C_{i_{3}}$, with $1<i_{2}<i_{1}<$ $i_{3}<m$. Continuing inductively, there can be at most 4 members of $\left\{C_{i}: 1<i<m\right\}$ having the form $B^{j-1} A$, with $j<n-2$, at most 8 members of $\left\{C_{i}: 1<i<m\right\}$ having the form $B^{j-1} A$ with $j<n-$ $3, \cdots$, and finally, at most $2^{n-2}$ members of $\left\{C_{i}: 1<i<m\right\}$ having the form $B^{j-1} A$ with $j<n-(n-2)=2$ (that is, $j=1$ and $B^{j-1} A=$ $A$ ). Consequently we can estimate the size of $U\left\{C_{i}: 1<i<m\right\}$. Analogous to the situation that, if $N(1, j) \cap N(x, k) \neq \varnothing$ for $1, x \in$ $G^{\sim}$, then for any $z_{1}, z_{2} \in N(1, j) \cup N(x, k)$ one has $d\left(z_{1}, z_{2}\right)<2 j+2 k$, there is the fact that if $B^{j} \cap B^{k} z \neq \varnothing$, then for any $z_{1}, z_{2} \in B^{j} \cup B^{k} z$, one has that $\left\{z_{1}\right\}$ and $\left\{z_{2}\right\}$ are not separated by at least $2 j+2 k$. Applying this fact to $U\left\{C_{i}: 1<i<m\right\}$, where each $C_{i}$ is of the form $B^{j-1} A$, which is contained in $B^{j} z$ for some $z$, we obtain that for any $z_{1}, z_{2} \in \cup\left\{C_{i}: 1<i<m\right\}$, the sets $\left\{z_{1}\right\}$ and $\left\{z_{2}\right\}$ are not separated by at least width

$$
2(n-1)+2 \cdot 2(n-2)+\cdots+2^{n-2} \cdot 2,
$$

where the $2(n-1)$ comes from the one $C_{i_{1}}$, where $2 \cdot 2(n-2)$ comes from the at most two $C_{i_{2}}$ and $C_{i_{3}}, \cdots$, and the $2^{n-2} \cdot 2$ comes from the at most $2^{n-2}$ members of $\left\{C_{i}: 1<i<m\right\}$ which were building blocks at stage 1. But routine use of inequalities shows that

$$
2(n-1)+2 \cdot 2(n-2)+\cdots+2^{n-2} \cdot 2<n\left(2^{n}-2\right)<4^{n}-2^{n+1} .
$$

Consequently for any $z_{1}$ and $z_{2} \in \cup\left\{C_{i}: 1<i<m\right\}$, we have that $\left\{z_{1}\right\}$ and $\left\{z_{2}\right\}$ are not separated by at least width $4^{n}-2^{n+1}$.

However, $\cup\left\{C_{i}: 1<i<m\right\}$ must "span the gap between $C_{1}$ and $C_{m}$," where $C_{1}=B^{n-1} A_{1}$ and $C_{m}=B^{n-1} A_{2}$. Using arguments similar to those above, one shows that $B^{n-1} A_{1}$ and $B^{n-1} A_{2}$ are separated by at least width $4^{n-1}-4-2(n-1)$, which is greater than $4^{n}$. Consequently two elements of $U\left\{C_{i}: 1<i<m\right\}$ must be separated by at least $4^{n}$, a contradiction. So Lemma 4 is proved.

To conclude the proof of (c), let $K$ be an arcwise connected, compact subset of $G$, and it will be shown that $i^{-1}(K)$ is compact in $G^{\sim}$. By using a translate, we assume $1 \in K$. The collection $\left\{W_{n}\right\}$ forms an open cover of $K$ by open sets in $G$, so a finite number of the collection $\left\{W_{n}\right\}$ cover $K$. So there exists $n$ such that $K \subset W_{n}$. Since $1 \in K$ and $K$ is arcwise connected, $K$ is contained in the arc 
component of $W_{n}$ at 1 . We now prove by induction that for all $n$, each arc component of $W_{n}$ is the union of a finite number of building blocks. It is clear for $n=1$, and we suppose it true for all $j<n$. Let $C$ be an arc component of $W_{n}$. In the case that $C$ contains no building block $B^{n-1} A$ obtained at stage $n$, then in fact $C \subset W_{j}$ for $j<n$ and our claim is true for $C$. By Lemma 4, $C$ does not contain two or more building blocks of the form $B^{n-1} A$. So we may suppose that $C$ contains exactly one $B^{n-1} A$. It can be shown that $C=B^{n-1} A \cup\left(\cup\left\{C_{t}\right\}\right)$, where each $C_{t}$ is an arc component in $W_{n-1}$, where $C_{t} \cap B^{n-1} A \neq \varnothing$, and where $C_{s} \cap C_{t}=\varnothing$ for $s \neq t$. In other words, $C$ is the union of all the arc components of $W_{n-1}$ which meet $B^{n-1} A$, along with $B^{n-1} A$. Since each $C_{t}$ is the union of a finite number of building blocks by the induction hypothesis, it suffices to show that $\left\{C_{t}\right\}$ is a finite collection. For each $C_{t}$, since $C_{t} \cap B^{n-1} A \neq \varnothing$, there exists $x$ and an open ball $B_{t}$ of radius 1 contained in $C_{t}$ satisfying $x \in B^{n-1} A \cap B_{t}$. The $\left\{B_{t}\right\}$ is a mutually disjoint collection, since the $\left\{C_{t}\right\}$ were such. Since $B^{n-1} A$ has compact closure in $G^{\sim}$ (recall that $B^{-}$was compact in $G^{\sim}$, that a translate of $A$ is contained in $B$, that $B^{n-1} A \subset\left(B^{-}\right)^{n-1} A^{-}$, and that $\left(B^{-}\right)^{n-1} A^{-}$ is the continuous image of a certain compact product space), there exists a subnet of $\left\{x_{t}\right\}$ converging to some $x \in G^{\sim}$. Denoting the subnet also by $\left\{x_{t}\right\}$, we have that $x_{t} x^{-1}$ converges to $1 \in G^{\sim}$, and each $x_{t} x^{-1} \in B_{t} x^{-1}$, with $\left\{B_{t} x^{-1}\right\}$ being a collection of mutually disjoint open balls of radius 1 . This can happen in a normal neighborhood of 1 in a Lie group only in the case that the collection is a finite collection. So the induction is finished. So $K$ is contained in the union of a finite number of building blocks. By the argument just given above, each building block has compact closure. Since $K$ is contained in a subset of $G^{\sim}$ having compact closure, and since $K=i^{-1}(K)$ is closed in $G^{\sim}$, we have that $i^{-1}(K)=K$ is compact in $G^{\sim}$, proving (c).

The next theorem discusses the existence of maximal compact subgroups of $G$.

THeORem 6. Let $G$ be arcwise connected and finite dimensional, and let $i: G^{\sim} \rightarrow G$. Then $G$ has maximal compact subgroups, and each one is of the form $i(K)$, where $K$ is a maximal compact subgroup of $G$. Consequently each such is a compact, connected Lie subgroup.

Proof. Use continuity of $i$, use (a) of the previous theorem, and use the results on the existence of maximal compact subgroups of Lie groups (for example [8, p. 180]). Other properties for $i(K)$ 
may be established by using the corresponding properties for $K$.

In the abelian case, $G^{\sim}$ has a unique maximal compact subgroup $K$ which is a direct factor in $G^{\sim}$. In fact, $G^{\sim}$ is isomorphic with $R^{n} \otimes K$. However, the author cannot answer the question whether $i(K)$ is a direct factor in $G$.

There are other results on certain subgroups of $G$; these results are suggested by surveying the known results on subgroups of Lie groups. Typical of these is the following theorem.

THEOREM 7. Let $G$ be arcwise connected and finite-dimensional, and let $i: G^{\sim} \rightarrow G$. Then there exists a unique maximal closed connected normal solvable subgroup $R$ of $G$. If $P$ denotes the radical of $G^{\sim}$, then $R=i(P)^{-}$and $R \subset Z(G) \cdot i(P)$. The subgroup $R$, although connected, may not be arcwise connected.

Proof. Define $R$ to be $i(P)^{-}$. Then clearly $R$ is a connected normal solvable subgroup of $G$. If $R_{1} \supset R$ and if $R_{1}$ has these properties, then, making use of $i^{-1}\left(R_{1}\right)$ and $i^{-1}(R)$ in $G^{\sim}$, it can be shown that $R_{1} / R$ is a connected, countable topological group (in fact abelian). Using the fact that $R_{1} / R$ is completely regular, $R_{1} / R$ is the oneelement group. The other conclusions follow routinely.

We now give some examples of arcwise connected, finite-dimensional groups in order to illustrate some limitations on the structure theorems which one might conjecture.

EXAMPLE 1. There exists a topology for $R^{n}$ such that $R^{n}$ is a topological group, $R^{n}$ is arcwise connected and finite-dimensional, and $R^{n}$ has no closed subgroups other than $\{0\}$ and $R^{n}$.

Proof. Nienhuys [11] and Rolewicz [12] have given a construction to obtain such a topology on $R^{1}$. We indicate how to alter their construction to obtain a topology on $R^{2}$ with the desired properties. It will then be clear how to obtain the topology for $R^{n}$. We will use the notation of Nienhuys [11]. In particular additive notation is used for groups in this example. The group $G$ is defined by Nienhuys to be a certain subgroup of $(R / Z)^{c}$, where $c$ is the countable cardinal. For $x \in(R / Z)^{c},\|x\|$ is defined to be lub $\left\{\left|x_{n}\right|\right.$ $\bmod Z: n \in N\}$. The metric for $G$ is given by $d(x, y)=\|x-y\|$. Also $P_{n}:(R / Z)^{c} \rightarrow(R / Z)^{c}$ is defined by $P_{n}(y)=\left(y_{1}, y_{2}, \cdots, y_{n}, 0,0, \cdots\right)$, where 0 is the identity of $R / Z$.

As Nienhuys does for $R^{1}$, we will construct a subgroup $A$ of $G$, and then embed $R^{2}$ in $G / A$. In fact, $A$ is the infinite cyclic sub- 
group of $G$ generated by $a \in G$, where $a$ is defined on p. 168 [11]. In order to embed $R^{2}$ in $G / A$, we pick inductively the irrational numbers $\lambda_{n}$ and $\gamma_{n}$ so that (1) $\left\{1, \lambda_{1}, \lambda_{2}, \cdots, \gamma_{1}, \gamma_{2}, \cdots\right\}$ is a linearly independent set over the rational numbers, (2) $\left|\gamma_{n}\right|<\left(2^{n} \cdot k_{n-1}\right)^{-1}$ and $\left|\lambda_{n}\right|<\left(2^{n} \cdot k_{n-1}\right)^{-1}$, where $k_{n}$ is the smallest integer such that for every $y \in G$ there exists some $k \leqq k_{n}$ satisfying

$$
\left\|k P_{n}(\lambda)-P_{n}(y)\right\| \leqq 1 / 2^{n} \text { and }\left\|k P_{n}(\gamma)-P_{n}(y)\right\| \leqq 1 / 2^{n} \text {, }
$$

(3) $\left|\lambda_{n+1}\right| \leqq 2^{-n}\left|\lambda_{n}\right|$ and $\left|\gamma_{n+1}\right| \leqq 2^{-n}\left|\gamma_{n}\right|$ for all $n \in N$, (4) if $p_{n}$ denotes the $n$th prime number, then $\lambda_{n}=r_{n}\left(p_{n}\right)^{1 / 9}$ and $\gamma_{n}=s_{n}\left(p_{n}\right)^{1 / 3}$, where $r_{n}$ and $s_{n}$ are rational numbers chosen inductively so that (1), (2), and (3) above hold. The existence of such $k$ follows from a version of Kronecker's approximation theorem (see [7], $\$ 26.19(\mathrm{~d})$, p. 436). The sequences $\left\{\lambda_{n}\right\}$ and $\left\{\gamma_{n}\right\}$ then determine elements $\lambda$ and $\gamma$ in $G$, by defining the $n$th coordinate of $\lambda$ to be $\lambda_{n}+Z$, similarly for $\gamma$. We define $f: R^{2} \rightarrow G$ by $f(r, s)=r \lambda+s \gamma$. It is clear that $f$ is a continuous homomorphism where $R^{2}$ has its usual topology and $G$ has the relative topology from $(R / Z)^{c}$. We show $f$ is one-one. Suppose $r \lambda+s \gamma$ is the identity of $G$ for some $r, s \in R$. Then for all $i \in N$ there exists $z_{i} \in Z$ such that $r \lambda_{i}+s \gamma_{i}=z_{i}$. So $r \lambda_{1}+s \gamma_{1}=z_{1}$ and $r \lambda_{2}+s \gamma_{2}=z_{2}$. The determinant of this system is not zero (it is $\left.\lambda_{1} \gamma_{2}-\lambda_{2} \gamma_{1}\right)$; so that, after solving for $r$ and $s$, we find that $r$, s belong to the subfield $F_{1}$ generated by $\left\{1, \lambda_{1}, \lambda_{2}, \gamma_{1}, \gamma_{2}\right\}$. In a similar manner, using the equations $r \lambda_{i}+s \gamma_{i}=z_{i}$, for $i=3$, 4, we find that $r, s$ belong to the subfield of the reals generated by $\left\{1, \lambda_{i}, \gamma_{i}: i=3,4\right\}$, where, from above, $\lambda_{i}=r_{i}\left(p_{i}\right)^{1 / 2}$ and $\gamma_{i}=s_{i}\left(p_{i}\right)^{1 / 3}$. Since $F_{1} \cap F_{2}$ is the rational numbers, $r$ and $s$ are rational numbers. However, using linear independence of the $\lambda$ 's and the $\gamma$ 's over the rationals, it follows that $r$ and $s$ are zero. So $f$ is one-one.

Next we let $p: G \rightarrow G / A$ be the coset homomorphism, and we show that $p$ restricted to $f\left(R^{2}\right)$ is one-one. We show that if $r \lambda+$ $s \gamma \in A$, then $r \lambda+s \gamma$ is the identity of $G$. Suppose that $r \lambda+s \gamma=$ $k a$, where $k$ is an integer. Then $r \lambda_{n}+s \gamma_{n}=k \cdot t_{n} / n \bmod Z$ for all $n$. Since $t_{n} \geqq 1 / 4$, we have that $\left|k t_{n}\right| n|\geqq| k \mid / 4 n$. On the other hand $\left|k t_{n}\right| n|=| r \lambda_{n}+s \gamma_{n}|\leqq| r|\cdot| \lambda_{n}|+| s|\cdot| \gamma_{n} \mid \bmod Z$. Furthermore, using property (3) above, we get $\left|\lambda_{n}\right| \leqq\left|\lambda_{1}\right| / 2^{s_{n}}$ and $\left|\gamma_{n}\right| \leqq\left|\gamma_{1}\right| / 2^{s_{n}}$, where $s_{n}$ is the sum of the first $n-1$ natural numbers. If $k$ is not 0 , then (using linear independence) at least one of $i$ and $j$ is not 0 , and therefore for large $n$ the two inequalities $\left|k t_{n}\right| n|\geqq| k \mid / 4 n$ and $\left|k t_{n}\right| n \mid \leqq 2^{-s_{n}}\left(\left|\boldsymbol{r} \lambda_{1}\right|+\left|s \gamma_{1}\right|\right) \bmod Z$ are both valid. This is clearly impossible, in as much as the sequence $\{1 / 4 n\}$ goes to zero much slower than the sequence $\left\{2^{-s_{n}}\right\}$. Therefore $k=0$, and $r \lambda+s \gamma$ is the identity of $G$. Consequently $p f$ is a one-one homomorphism and 
can be used to define a topology on $R^{2}$. Since $p f$ is continuous, it follows that this topology is arcwise connected and finite-dimensional.

Next we show that $R^{2}$ has no closed subgroups other than the identity and $R^{2}$. We do this by proving that for all $(r, s) \in R^{2}$, with $(r, s) \neq(0,0)$, the subgroup generated by $(r, s)$ is dense in $R^{2}$. So let $d=r \lambda+s \gamma \in f\left(R^{2}\right)$. To show $d$ generates a dense subgroup of $G$, we let $\varepsilon$ be any positive real number and let $y \in G$. We imitate the proof of [11] with minor modifications. From the definition of $G$, there is a natural number $N$ such that for all $n>N, \| P_{n}(y)-$ $y \|<\varepsilon / 3$. Let $c=|r|+|s|$. Let us first suppose that $r$ and $s$ are both different from 0 . For every $n$, there exists $k \leqq k_{n}$ such that $\left\|k P_{n}(\lambda)-P_{n}(y / 2 r)\right\| \leqq 1 / 2^{n}$, that $\left\|k P_{n}(\lambda)-P_{n}(y / 2 s)\right\| \leqq 1 / 2^{n}$, that $\left|\lambda_{n}\right|<$ $1 / 2^{n} \cdot k_{n-1}$, and that $\left|\gamma_{n}\right|<1 / 2^{n} k_{n-1}$. Then we have that $\| k P_{n}(d)-$ $P_{n}(y)\|\leqq\| k P_{n}(r \lambda+s \gamma)-P_{n}(y / 2)-P_{n}(y / 2)\|\leqq\| k P_{n}(r \lambda)-P_{n}(y / 2) \|+$ $\left.\left\|k P_{n}(s \gamma)-P_{n}(y / 2)\right\|=|r| \cdot \| k P_{n}(\lambda)-P_{n}(y / 2 r)\right)\|+|s| \cdot\| k P_{n}(\gamma)-P_{n}(y \mid$ $2 s) \| \leqq|r| / 2^{n}+|s| / 2^{n}=c / 2^{n}$. Consequently, there is a natural number $M$ such that for all $n>M$, $\left\|k P_{n}(d)-P_{n}(y)\right\| \leqq c / 2^{n}<\varepsilon / 3$. Also note that for any $n>M$, we have $|k| \cdot\left|d_{n}\right|=|k| \cdot\left|\boldsymbol{r} \lambda_{n}+s \gamma_{n}\right| \leqq(|k| c) \mid$ $2^{n+1} k_{n} \leqq\left(c / 2^{n+1}\right)\left(|k| / k_{n}\right) \leqq c / 2^{n+1}<\varepsilon / 3$. Hence for such $n, \| k P_{n}(d)-$ $k d \|<\varepsilon / 3$. So we see that $\|k d-y\| \leqq\left\|k d-k P_{n}(d)\right\|+\| k P_{n}(d)-$ $P_{n}(y)\|+\| P_{n}(y)-y \|<3 \cdot(\varepsilon / 3)$ for sufficiently large $n$. A similar proof is made in the case that one of $i$ or $j$ is zero. Consequently we have shown that the group generated by $(r, s)$ is dense $R^{2}$ (it is easily shown that $p f(r, s)$ is dense in $G / A$ once one has shown that $f(r, s)$ is dense in $G)$.

In the case for constructing a topology for $R^{n}, n>2$, we indicate some of the changes needed to alter the above proof for $R^{2}$.

(a) Instead of determining $\lambda$ and $\gamma$ in $G$, one determines $n$ elements $\delta_{1}, \cdots, \delta_{n}$ in $G$ so that similar statements to $(1)-(4)$ hold. For example, in (1), all coordinates of $\delta_{i}$ for all $i$ must form a linearly independent collection of irrational numbers; in (2) and (3) instead of two inequalities there are $n$ inequalities; and in (4) $\left(\delta_{1}\right)_{k}=$ $r_{k}\left(p_{k}\right)^{1 / 2},\left(\delta_{2}\right)_{k}=r_{2 k}\left(p_{k}\right)^{1 / 3}, \cdots$, and $\left(\delta_{n}\right)_{k}=r_{n k}\left(p_{k}\right)^{1 / p_{n}}$.

(b) Instead of defining $f$ as above, one defines $f: R^{n} \rightarrow G$ by $f\left(t_{1}, \cdots, t_{n}\right)=t_{1} \delta_{1}+\cdots+t_{n} \delta_{n}$.

(c) Instead of knowing that the determinant is $\lambda_{1} \gamma_{2}-\lambda_{2} \gamma_{1}$, which is not zero, one notes that the determinant of the matrix $\left[\left(\delta_{i}\right)_{j}\right]$, with $1 \leqq i, j \leqq n$, is not zero.

(d) Instead of obtaining two subfields $F_{1}$ and $F_{2}$ of the real numbers, one obtains $n$ subfields whose intersection is the rational numbers.

DEFinition. The topology obtained above for $R^{k}$ will be called the $N$-topology (for no nontrivial closed subgroups). Since $Z^{k}$ is a 
subgroup of $R^{k}$, the relative topology for $Z^{k}$ obtained from the $N$ topology on $R^{k}$ will be called the $M$-topology. Note that with the $M$-topology, $Z^{k}$ has no nontrivial closed subgroups.

Next we use Example 1 to aid in the construction of other examples. One version of Weil's lemma states that if $G$ is a locally compact group, and if $P$ is a one-parameter subgroup of $G$, then either $P$ is isomorphic to $R$, or $P^{-}$is compact.

EXAMPLE 2. There is an arcwise connected, finite-dimensional group $G$ with a one-parameter subgroup $P$ such that $P$ is not isomorphic to $R$, such that $P^{-} \neq P$, and such that $P^{-}$is not compact. Also $P^{-}$is not arcwise connected (although $P$ is connected).

Proof. Let $Z^{2}$ have a $M$-topology $T$ given in the above definition. For each open neighborhood $B=N(0, \delta)$ in $R^{2}$ with its usual metric $d$, where 0 is the identity of $R^{2}$, and where $\delta>0$, and for each $D \in T$, we define $W(D, \delta)=D+B=\{d+x: d \in D$ and $x \in B\}$. Then the set of all such $D+B$ forms a neighborhood base $\mathscr{B}$ at $0 \in R^{2}$ for a topology $U$ making $G=\left(R^{2}, U\right)$ into an arcwise connected, two-dimensional topological group. Note that $\mathscr{B}$ is of the type described in Theorem 1 . Also $Z^{2}$ is a closed subgroup of $G$, and $Z^{2}$ is a minimal member of $\mathscr{S}$ in the sense of Theorems 3, 4. Let $I=$ $\{(x, 0): 0 \leqq x \leqq 1\}$. Then $I$ is a compact subset of $G$. Hence $Z^{2}+I$ is a closed subset of $G$. But $Z^{2}+I=R \times Z$. Hence $R \times Z=(R \times\{0\})^{-}=$ $P^{-}$. Then $P$ is a one-parameter subgroup such that $P$ meets the desired conditions.

For a locally compact group $G$ with one-parameter subgroup $P$, the subgroup $P^{-}$has either one or uncountably many arc components. Note that $P^{-}$in Example 2 has a countably infinite number of arc components.

EXAMPLE 3. There is an arcwise connected, finite-dimensional group $G$ with a one-parameter subgroup $P$ such that $P$ is not isomorphic with $R$, such that $P^{-} \neq P$, and such that $P^{-}$is not compact. Also $P^{-}$is arcwise connected.

Proof. Let $R$ have a $N$-topology $T$; and let $H=(R, T)$, in order to distinguish the two different topologies for $R$. Let $C=$ $\{\exp (i r): 0 \leqq r \leqq 2 \pi\}$ be the usual circle group. Then define $G=C \otimes$ $H$, where $G$ has the product topology. Define the one-parameter subgroup $P \subset G$ by $f: R \rightarrow G$ where $f(r)=(\exp (i r), r)$ for $r \in R$. We will prove that $P^{-}=G$. Note that $\{(\exp (0), 2 \pi n): n$ is an integer $\}$ is a subgroup of $P \cap(\{\exp (0)\} \times H)$. So the closure of $\{(\exp (0)$, 
$2 \pi n): n$ is an integer $\}=\{\exp (0)\} \times H$. So $P \cup\{\exp (0)\} \times H \subset P$. So $G \subset P^{-}$. (The symbol $\otimes$ means direct product).

Note that in this example $G$ has a nontrivial compact Lie subgroup. Another example meeting the conditions of this Example 3 could be given. Let $G$ be $R^{2}$ with a $N$-topology, and let $P=\{0\} \times$ $R$. Then $P^{-}=R^{2}=G$.

We now give an example of a group $G$ as mentioned in Theorem 7 .

Example 4. There exists a $G$ as in Theorem 7 whose radical $R$ is not arcwise connected.

Proof. Let $S$ be a simple Lie group whose center $Z$ is isomorphic to the integers, and let $w$ be a generator of $Z$. We let $A=$ $P^{-}$, where $P^{-}$is given in Example 2, and $P^{-}=R \times Z$ as sets. We let $D$ be the subgroup of $S \otimes A$ generated by $(w,(0,1))$. Then $D$ is a discrete central subgroup of $S \otimes A$, discreteness following from the facts that $w$ generates a discrete subgroup of $S$ and the topology for $S \otimes A$ is the product topology. Then we define $G=$ $(S \otimes A) / D$. Note that $S \otimes A$ is not arcwise connected, because $A$ is not; however $(S \otimes A) / D$ is arcwise connected. Also $(Z \otimes A) / D$ is the $R$ of Theorem 7 , and $(Z \otimes A) / D$ is not arcwise connected. In fact, $(Z \otimes A) / D$ is isomorphic to $A$.

\section{REFERENCES}

1. Haskell Cohen, A cohomological definition of dimension, Duke Math. J., 21 (1954), 209-224.

2. C. H. Dowker, Inductive dimension of completely normal spaces, Quat. J. Math. (Oxford), 4 (1953), 267-281.

3. J. Dugundji, Topology, Allyn and Bacon, Boston, 1966.

4. A. Gleason and R. Palais, On a class of transformation groups, Amer. J. Math., 79 (1957), 631-648.

5. M. Goto, Dense embeddings of locally compact connected groups, Ann. Math., 61 (1955), 154-169.

6. S. Helgason, Differential Geometry and Symmetric Spaces, Academic Press, 1962.

7. E. Hewitt and K. Ross, Abstract Harmonic Analysis, vol. 1, Springer Verlag, Berlin, 1963.

8. G. Hochschild, The Structure of Lie Groups, Holden-Day, Inc., San Francisco, 1965.

9. Karl H. Hofmann and P. Mostert, Elements of Compact Semigroups, Merrill, Columbus, Ohio, 1966.

10. S. N. Hudson, On connectivity properties of finite-dimensional groups, P.A.M.S., 23 (1969), 68-72.

11. J. Nienhuys, A solenoidal and monothetic minimally almost periodic group, Fund. Math., 73 (1971), 167-169.

12. S. Rolewicz, Some remarks on monothetic groups, Coll. Math., 13 (1964), 27-28. 
Received March 13, 1978 and in revised form July 17, 1978. This research was partially supported by the U. S. National Science Foundation under Grant HES7306184. The author is grateful to the Mathematics Department of Dartmouth College for the use of their facilities.

Savannah State College

SAVANNAH, GA 31404 


\section{PACIFIC JOURNAL OF MATHEMATICS}

\section{EDITORS}

Donald BABBITT (Managing Editor)

University of California

Los Angeles, CA 90024

HUGo RossI

University of Utah

Salt Lake City, UT 84112

C. C. MOORE and ANDREW OGG

University of California

Berkeley, CA 94720

\section{J. DuGUNDJI}

Department of Mathematics

University of Southern California

Los Angeles, CA 90007

R. FINN and J. Milgram

Stanford University

Stanford, CA 94305

ASSOCIATE EDITORS
E. F. BECKENBACH
B. H. NeumanN
F. WOLF
K. YoSHIDA

\section{SUPPORTING INSTITUTIONS}

\author{
UNIVERSITY OF BRITISH COLUMBIA \\ CALIFORNIA INSTITUTE OF TECHNOLOGY \\ UNIVERSITY OF CALIFORNIA \\ MONTANA STATE UNIVERSITY \\ UNIVERSITY OF NEVADA, RENO \\ NEW MEXICO STATE UNIVERSITY \\ OREGON STATE UNIVERSITY \\ UNIVERSITY OF OREGON
}

\author{
UNIVERSITY OF SOUTHERN CALIFORNIA \\ STANFORD UNIVERSITY \\ UNIVERSITY OF HAWAII \\ UNIVERSITY OF TOKYO \\ UNIVERSITY OF UTAH \\ WASHINGTON STATE UNIVERSITY \\ UNIVERSITY OF WASHINGTON
}

The Supporting Institutions listed above contribute to the cost of publication of this Journal, but they are not owners or publishers and have no responsibility for its content or policies.

Mathematical papers intended for publication in the Pacific Journal of Mathematics should be in typed form or offset-reproduced, (not dittoed), double spaced with large margins. Please do not use built up fractions in the text of the manuscript. However, you may use them in the displayed equations. Underline Greek letters in red, German in green, and script in blue. The first paragraph or two must be capable of being used separately as a synopsis of the entire paper. Please propose a heading for the odd numbered pages of less than 35 characters. Manuscripts, in triplicate, may be sent to any one of the editors. Please classify according to the scheme of Math. Reviews, Index to Vol. 39. Supply name and address of author to whom proofs should be sent. All other communications should be addressed to the managing editor, or Elaine Barth, University of California, Los Angeles, California, 90024.

50 reprints to each author are provided free for each article, only if page charges have been substantially paid. Additional copies may be obtained at cost in multiples of 50 .

The Pacific Journal of Mathematics is issued monthly as of January 1966. Regular subscription rate: $\$ 84.00$ a year (6 Vols., 12 issues). Special rate: $\$ 42.00$ a year to individual members of supporting institutions.

Subscriptions, orders for numbers issued in the last three calendar years, and changes of address should be sent to Pacific Journal of Mathematics, P.O. Box 969, Carmel Valley, CA 93924, U.S.A. Older back numbers obtainable from Kraus Periodicals Co., Route 100, Millwood, NY 10546.

PUBLISHED BY PACIFIC JOURNAL OF MATHEMATICS, A NON-PROFIT CORPORATION

Printed at Kokusai Bunken Insatsusha (International Academic Printing Co., Ltd.). 8-8, 3-chome, Takadanobaba, Shinjuku-ku, Tokyo 160, Japan.

Copyright (C) 1979 by Pacific Journal of Mathematics Manufactured and first issued in Japan 


\section{Pacific Journal of Mathematics}

\section{Vol. 82 , No. 2 \\ February, 1979}

Krishnaswami Alladi and Paul Erdős, On the asymptotic behavior of large prime

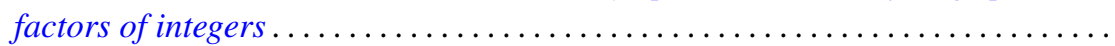

Alfred David Andrew, A remark on generalized Haar systems in $L_{p}$,

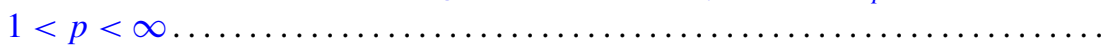

John M. Baker, A note on compact operators which attain their norm . . ........

Jonathan Borwein, Weak local supportability and applications to

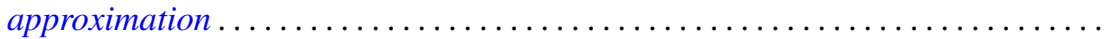

Tae Ho Choe and Young Soo Park, Wallman's type order compactification ........

Susanne Dierolf and Ulrich Schwanengel, Examples of locally compact

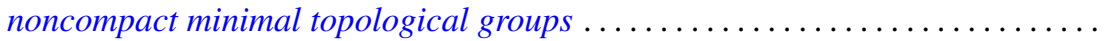

Michael Freedman, A converse to (Milnor-Kervaire theorem) $\times R$ etc. . . . . . . .

George Golightly, Graph-dense linear transformations ..................

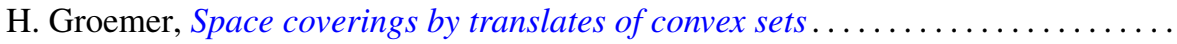

Rolf Wim Henrichs, Weak Frobenius reciprocity and compactness conditions in

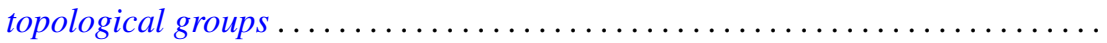

Horst Herrlich and George Edison Strecker, Semi-universal maps and universal

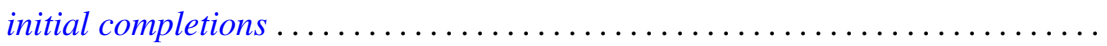

Sigmund Nyrop Hudson, On the topology and geometry of arcwise connected,

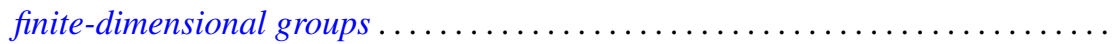

K. John and Václav E. Zizler, On extension of rotund norms. II .............

Russell Allan Johnson, Existence of a strong lifting commuting group of transformations. II.

Bjarni Jónsson and Ivan Rival, Lattice varieties covering the smallest nonmodular variety

Grigori Abramovich Kolesnik, On the order of Dirichlet L-functions .

Robert Allen Liebler and Jay Edward Yellen, In search of nonsolvable groups of

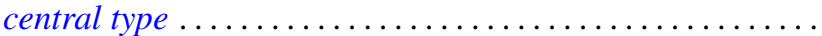

Wilfrido Martínez T. and Adalberto Garcia-Maynez Cervantes, Unicoherent plane Peano sets are $\sigma$-unicoherent ...

M. A. McKiernan, General Pexider equations. I. Existence of injective

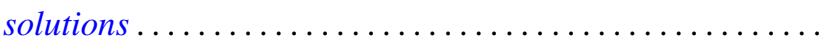

M. A. McKiernan, General Pexider equations. II. An application of the theory of webs.

Jan K. Pachl, Measures as functionals on uniformly continuous functions . .

Lee Albert Rubel, Convolution cut-down in some radical convolution algebras ...

Peter John Slater and William Yslas Vélez, Permutations of the positive integers

with restrictions on the sequence of differences. II . . .

Raymond Earl Smithson, A common fixed point theorem for nested spaces ....

Indulata Sukla, Generalization of a theorem of McFadden .... . . .

Jun-ichi Tanaka, A certain class of total variation measures of analytic measures.

Kalathoor Varadarajan, Modules with supplements .............. 